\title{
EFFECT OF NONSTOICHIOMETRIC DEFECTS AND Cr IONS ON THE PHOTOCONDUCTIVITY OF $\mathrm{Bi}_{12} \mathrm{SiO}_{20}$ CRYSTALS
}

\author{
T.V. Panchenko ${ }^{*}$, L.M. Karpova ${ }^{2}$ \\ ${ }^{1}$ Oles Honchar Dnipro National University, Dnipro, Ukraine \\ ${ }^{2}$ Ukrainian State University of Chemical Technology, Dnipro, Ukraine \\ e-mail: panchtv141@gmail.com
}

\begin{abstract}
The results of an experimental study of the effect of nonstoichiometric defects in oxygen and the $\mathrm{Bi}: \mathrm{Si}$ ratio, as well as $\mathrm{Cr}$ ions, on the photoconductivity of $\mathrm{Bi}_{12} \mathrm{SiO}_{20}$ crystals are presented. It is shown that varying the annealing conditions allows one to modify the spectral distribution and quantitative characteristics of photoconductivity substantially.
\end{abstract}

Keywords: surface photoconductivity, annealing, vacuum, silicosillenite crystals, chromium impurity.

Received 05.07.2020; Received in revised form 12.08.2020; Accepted 04.09.2020

\section{Introduction}

Crystals of the $\mathrm{Bi}_{12} \mathrm{MO}_{20}$ family of sillenites (BMO, where $\mathrm{M}=\mathrm{Si}, \mathrm{Ge}, \mathrm{Ti}$ ) have a unique set of practically useful properties (photorefractive, electro- and magneto-optical, piezoelectric and a number of other effects). They are successfully used in many areas of functional electronics [1]. Recently, designs of new functional devices in the sizes of nanoand micrometric scales have been being developed. In this connection, interest in obtaining and studying the properties of surface layer [2], micro- and nanocrystals of BMO [3], and also crystals of BMO with nanoscale inclusions [4] is increasing significantly. The task of modifying the properties of sillenites in thin layers near the surface is very actual.

A feature of BMO crystals is high photosensitivity in the visible wavelength range $\left(\sim 10^{-6} \mathrm{~J} \cdot \mathrm{cm}^{-2}\right)$. It is of interest to study the possibilities of a controlled modification of this sensitivity in the surface layers of BMO crystal samples. This paper presents the results of an experimental study of the effect of nonstoichiometric defects such as oxygen deficiency and variation of the $\mathrm{Bi}: \mathrm{Si}$ ratio in undoped $\mathrm{Bi}_{12} \mathrm{SiO}_{20}$ (BSO) crystals, as well as the effect of chromium impurities in doped $\mathrm{Bi}_{12} \mathrm{SiO}_{20}: \mathrm{Cr}$ (BSO:Cr) crystals.

\section{Experiment}

Undoped BSO crystals of stoichiometric composition and with non-stoichiometric defects in the form of deficiency ( $\mathrm{BSO}-\mathrm{Si}$ ) and excess silicon $(\mathrm{BSO}+\mathrm{Si})$, as well as $\mathrm{BSO}: \mathrm{Cr}$ crystals with different concentrations of $\mathrm{Cr}$ impurities were grown by the Czochralski method. The content of $\mathrm{Cr}$ ions in BSO:Cr crystals was determined by the method of spectral emission analysis and amounted to $\sim 10^{-4}, 10^{-3}, 10^{-2}$ and $10^{-1}$ mass. $\%$. Varying non-stoichiometry with respect to Si made it possible to vary the content of defects in the oxygen sublattice of tetrahedra, in the centers of which $\mathrm{Si}$ ions are located in BSO crystals.

The Cr impurity was chosen as the dopant due to its strong influence on the optical absorption and photochromic effect in sillenites.

Surface photoconductivity was studied in the spectral range of the energy of light quanta $h v=0.4-3.4 \mathrm{eV}$ at room temperature. We used a prism monochromator SPM with a $600 \mathrm{~W}$ xenon lamp. The light beam was modulated at a frequency of $12 \mathrm{~Hz}$. The measurements were carried out in a constant electric field, a stabilized voltage of $15 \mathrm{~V}$ was applied. The samples were prepared in the form of polished plates cut in the crystallographic plane (001). The useful signal was recorded using the synchronous detection technique. Ag electrodes with a gap between them of $1 \mathrm{~mm}$ were deposited on one of these planes. The useful signal was recorded using the synchronous detection technique. 
The dependences of the photocurrent $I^{P h}(h v)$ were normalized with respect to the spectral distribution of the flux of light quanta. The light intensity in the studied spectral range was brought to the same value using a set of neutral filters. Annealing the samples in vacuum $\sim 10^{-3} \mathrm{~Pa}$ was carried out at $\mathrm{T} \approx 830-850 \mathrm{~K}$ for 2 hours.

The measurement results are presented in the form of spectral dependences of the relative photoconductivity $\sigma^{P h}=\left(\sigma_{1}-\sigma_{0}\right) / \sigma_{0}$, where $\sigma_{1}$ and $\sigma_{0}$ are the surface conductivities during illumination and in the dark, respectively, on the energy of light quanta in the semi-logarithmic scale $L g \sigma^{P h}(h v)$.

\section{Experimental results and discussion}

BSO crystals are wide-gap semiconductors (band gap $\Delta \mathrm{E}_{\mathrm{g}} \approx 3.3 \mathrm{eV}$ at room temperature). The forbidden zone has a complex structure of local levels [5]. It is due to the high concentration $\left(\sim 10^{18} \mathrm{~cm}^{-3}\right)$ of intrinsic point defects. The most characteristic of them are non-stoichiometry defects. The stoichiometric composition contains 14.3 mol. \% $\mathrm{SiO}_{2}$ and $85.7 \mathrm{~mol} \% \mathrm{Bi}_{2} \mathrm{O}_{3}$. We made a $\mathrm{SiO}_{2}$ deficit $(10 \mathrm{~mol}$. \%) and an excess of $\mathrm{SiO}_{2}\left(17 \mathrm{~mol}\right.$. \%) due to the corresponding excess or deficiency of $\mathrm{Bi}_{2} \mathrm{O}_{3}$ in the charge. BSO crystals of stoichiometric composition were grown, as well as crystals with excess $(\mathrm{BSO}+\mathrm{Si})$ and silicon deficiency $(\mathrm{BSO}-\mathrm{Si})$.

The obtained dependences $L g \sigma^{P h}(h v)$ have the same character of the spectral distribution of photoconductivity with a wide intense weakly structured domed peak in the region $h v_{1}=2.1-3.1 \mathrm{eV}$ and a weak stepwise increase in photoconductivity in the region $h v_{2}=1.0-2.1 \mathrm{eV}$ (Fig. 1). In this case, before annealing in vacuum, the photoconductivity in both spectral regions $h v_{1}$ and $h v_{2}$ exceeds (BSO + Si crystals) or less (BSO - Si crystals) than the photoconductivity of BSO samples with stoichiometric composition (Fig. 1, a).

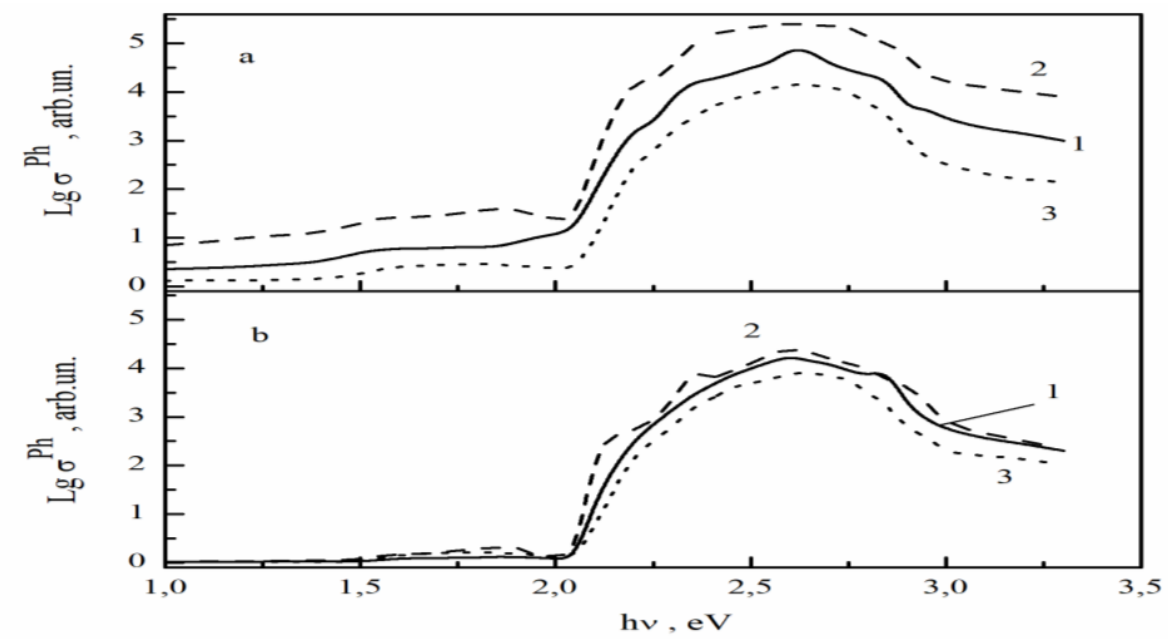

Fig. 1. The spectral dependences of photoconductivity of BSO (a, 1; b, 1), BSO + Si (a, 2; b, 2) and BSO - Si (a, 3; b, 3) crystals before (a) and after annealing in vacuum (b).

Thus, photoconductivity is associated with the defectiveness of the Si sublattice. It is known that in BSO crystals there are a few vacancies (up to 10\%) in $\mathrm{Si}^{4+}$ sites, which can be replaced by $\mathrm{Bi}^{3+}$ and $\mathrm{Bi}^{5+}$ ions. Such substitutions cause the appearance of acceptor and donor levels in the forbidden zone, respectively. To some extent, donor levels can be compensated. The filling of $\mathrm{Si}$ vacancies specified by nonstoichiometry in $\mathrm{BSO}+\mathrm{Si}$ 
crystals leads to a decrease in the degree of compensation and, consequently, to an increase in photoconductivity. We have the opposite situation for BSO - Si crystals.

Annealing in vacuum causes a decrease in photoconductivity and eliminates the difference in its value for BSO and BSO \pm Si crystals (Fig. 1, b). This is partly explained as follows. The appearance of oxygen vacancies favors the formation of hole centers of $\mathrm{O}^{-}$and nanosized complexes of the $\mathrm{Si}-\mathrm{O}-\mathrm{Si}$ type. They are characterized by intracenter absorption in the near UV region. Such absorption was observed in BSO crystals annealed in vacuum [6].

A possible reason for the extinction of photoconductivity may be the appearance of new recombination channels. 2-center recombination, for example, demonstrates itself in the phenomena of thermal activation and quenching of photoconductivity in BSO crystals [7].

The interest in studying the photoconductivity of BSO crystals doped with multiply charged chromium ions is due to the possibility of controlling their photoelectric properties and optical absorption due to the photochromic effect. This can be used, for example, in the development of planar optical waveguides with adjustable parameters.

The surface photoconductivity spectra $L g \sigma^{P h}(h v)$ of BSO:Cr crystals containing different concentrations of $\mathrm{Cr}$ ions obtained before and after annealing in vacuum are shown in Fig. 2 a, b.

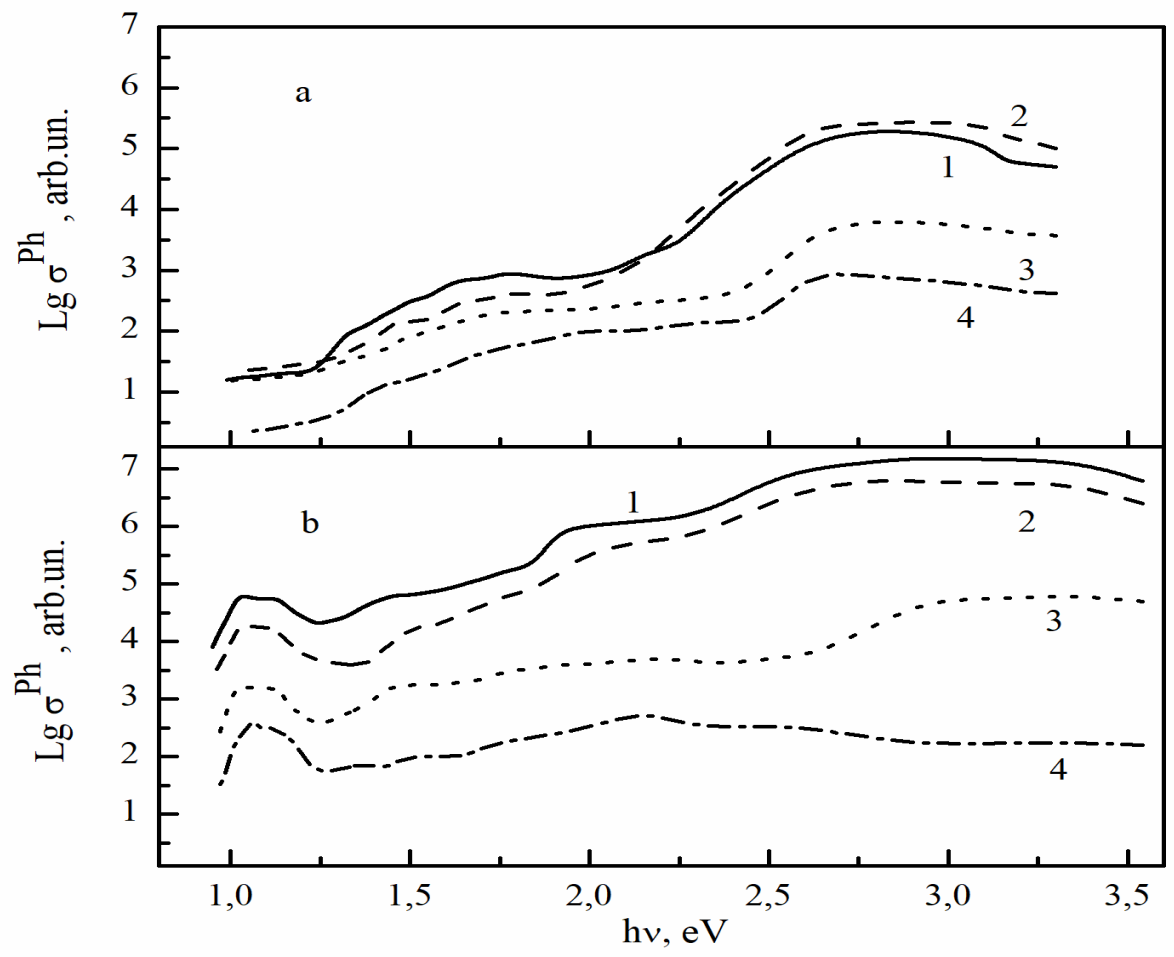

Fig. 2. The spectral dependences of photoconductivity of BSO: $\mathrm{Cr}$ crystals with a concentration of $\mathrm{Cr} 10-4(a, 1 ; b, 1), 10-3(a, 2 ; b, 2), 10-2(a, 3 ; b, 3)$ and 10-1 mass. \% (a, 4; b, 4) before (a) and after annealing in vacuum (b).

With an increase in the $\mathrm{Cr}$ content, a decrease in photosensitivity is observed in the entire studied spectral range. The nature of the spectral distribution of the photoresponse differs significantly from the photoresponse of undoped BSO with a more pronounced structure. 
The effect of vacuum annealing is opposite to the effect on undoped BSO photoconductivity does not decrease but grows within the order of magnitude over the entire studied range.

\section{Conclusions}

1. Varying the concentration of non-stoichiometry defects with respect to the $\mathrm{Si}: \mathrm{Bi}$ ratio when growing BSO crystals allows one to vary the surface photoconductivity within one (range $h v \approx 1-2 \mathrm{eV}$ ) and two (range $h v \approx 2-3.3 \mathrm{eV}$ ) orders of magnitude. If it is necessary to reduce the spatial heterogeneity of the distribution of defects of nonstoichiometry with respect to the $\mathrm{Si}: \mathrm{Bi}$ ratio and the related inhomogeneity of photoconductivity, the crystals should be annealed in vacuum.

2. Doping BSO crystals with $\mathrm{Cr}$ ions allows one to change the spectral distribution of photoconductivity and increase it within an order of magnitude in the region with $h v<2 \mathrm{eV}$, however, with increasing concentration, the photoconductivity decreases.

Vacuum annealing provides an increase in photoconductivity in the entire spectral range with the appearance of a peak in the region $h v \approx 0.9-1.4 \mathrm{eV}$.

\section{References}

1. Gunter, P. Photorefractive Materials and Their Applications. Part.1 / P. Gunter, J. Huignard // Springer Science+Business Media, New York. - 2006. - doi.org/10.1007/b106782.

2. Veber A. Dielectric Properties of Sol-Gel-Derived $\mathrm{Bi}_{12} \mathrm{SiO}_{20}$ Thin Films / A. Veber, S. Kunej, D. Suvorov // J. Am. Ceram. Soc. - 2013. - Vol. 96, No. 1. - P. 157 - 160. - doi: 10.1111/j.1551-2916.2012.05438.X.

3. Wan, Zhen. Controlled synthesis and visible light photocatalytic activity of $\mathrm{Bi}_{12} \mathrm{GeO}_{20}$ uniform microcrystals / Zhen Wan \& Gaoke Zhang // Scientific Reports. 2014. - Vol. 4. - P. 6296 - 6298. doi: 10.1038/srep06298.

4. Filipič, C. Dielectric relaxation in pure and Co-doped $\mathrm{Bi}_{12} \mathrm{GeO}_{20}$ single crystals / C. Filipic, A. Klos, M. Gajc, D.A. Pawlak, J. Dolinšek, A. Levstik // Journal of Advanced Dielectrics. - 2015. - Vol. 5, No. 3. - P.15500223-1 - 5. doi: 10.11142/S20101.35X1550023X.

5. Malinovsky, V.K. Photoinduced phenomena in sillenites / V.K. Malinovsky, O.A Gudaev, S.I. Gusev, V.A. Demenko. - Novosibirsk: Nauka, 1990 (in Russian).

6. Khudyakova, E.S. The effect of annealing on the spectral dependences of the opticalabsorption in sillenite crystals. / E.S. Khudyakova, F.N. Grebenchukov // Bulletin of the Samara Scientific Center of the Russian Academy of Sciences. - 2013. - Vol. 15, No. 4. $-136 \mathrm{~K}-139 \mathrm{~K}$.

7. Panchenko, T.V. Photoelectric properties of $\mathrm{Bi}_{12} \mathrm{SiO}_{20}$ crystals / T.V. Panchenko, Z.Z Yanchuk // Phys. Stat. Sol. -1996. - Vol. 38, No. 7. - P. 2018 - 2028. 